\title{
Morphological and functional changes of the optic nerve following traumatic optic nerve injuries in rabbits
}

\author{
FEI XUE, KUNMING WU, TIANYOU WANG, YOU CHENG, MANJIE JIANG and JUNFENG JI \\ Department of Otolaryngology and Head Neck Surgery, Jinling Hospital, Nanjing, Jiangsu 210002, P.R. China
}

Received November 5, 2015; Accepted December 18, 2015

DOI: $10.3892 /$ br.2016.567

\begin{abstract}
The aim of the present study was to investigate the morphological changes of the optic nerve following traumatic injuries and decompression at different times after injury, and to observe the changes of the visually evoked potentials, to identify the relevant associations between surgical opportunity and the clinical effect of traumatic optic nerve injuries. Rabbits were chosen as the animal model for the study. All the rabbits were randomly divided into five groups (A-E), representing the normal control, decompression in $48 \mathrm{~h}$, in 1 week, in 2 weeks and non-decompression groups, respectively. The pattern reversal visual evoked potentials (P-VEP) and morphological changes of the optic nerve were observed. The P-VEP of each healthy rabbit revealed typical NPN contours, while NPN waves in the injured rabbits were low and flat. The latent period of the P-wave was lengthened and the amplitude was reduced. The differences of the latent period and amplitude pre- and post-trauma were statistically significant. The morphological changes were also assessed. In the normal control group, the astrocytes of the optic nerve exhibited a cylindrical form and were arranged evenly on the vertical section. The neural fibers were arranged neatly, were even following application of a dye, and the cross section exhibited a normal configuration of the blood vessel. For the 48-h decompression group, the arrangement of the astrocytes was even on the vertical section, and vacuoles, slight swelling of the nerve, exudation around the blood vessel and a small amount of astrocytic hyperplasia were observed in the damaged area. In the non-decompression group there were large areas of necrosis, clear nerve demyelination, serious exudation around the blood vessel and astrocytic hyperplasia were observed. In conclusion, the optic nerve decompression is beneficial to protect the visual function in indirect optic nerve injuries. Visual function may be improved by decompression in $48 \mathrm{~h}$ compared to 2 weeks. In order to
\end{abstract}

Correspondence to: Dr You Cheng, Department of Otolaryngology and Head Neck Surgery, Jinling Hospital, 305 East Zhongshan Road, Nanjing, Jiangsu 210002, P.R. China

E-mail: chengyou2002@126.com

Key words: animal model, optic nerve injury, visual evoked potentials, pathology prevent secondary axon injury and to protect visual functions, the decompression should be performed as soon as possible.

\section{Introduction}

Traumatic optic nerve injury is one of the complications of head trauma, and is also the main cause of head trauma causing permanent vision loss. The optic nerve is located at the back of the eyes. Head or orbital trauma can cause optic canal fracture fragments, which may cut off or oppress the optic nerve. Optic nerve sheath membrane hemorrhage due to the fracture of the skull base can cause subdural or subarachnoid hemorrhage, and optic nerve avulsion can be caused by orbital trauma. All these can cause traumatic optic nerve injury (1). Visual evoked potential examination has an extremely important value for the diagnosis of traumatic optic neuropathy. Currently, it is a widely used clinical objective evaluation method for visual function following injury. In the present study, through an established animal model of indirect optic nerve injury, visual evoked potential and the morphological changes of the optic nerve tissue were observed following injury, as well as the decompression changes at different times of the trauma model, in order to study the mechanism of traumatic optic nerve injury and the association between the surgical time and curative effect, to offer a theoretical foundation for the selection of a suitable surgical time for clinical treatment of traumatic optic neuropathy.

\section{Materials and methods}

Experimental materials and groups. A total of 30 healthy adult male New Zealand white rabbits, with a body weight of 2.0-3.0 kg, were provided by the Animal Experiment Center of Nanjing General Hospital of Nanjing Command (Nanjing, China). Upon examination, all rabbits had normal refractive media, equal and round pupils, and normal reaction to light, without fundus oculi abnormalities. All the rabbits were randomly divided into five groups (A-E), with 6 rabbits in each group, representing the normal control, 48-h decompression, 1-week decompression, 2-week decompression (1) and non-decompression groups, respectively. The left eye of each animal was selected for analysis.

Establishment of the optic nerve crush injury model. Rabbits were anesthetized by subcutaneous administration of $50 \mathrm{~g} / 1$ ketamine $(100 \mathrm{mg} / \mathrm{kg})$. The Tenon' s capsule was incised from 
the supraorbital rim, the superior and medial rectus were separated and clipped, and the optic foramen was separated and exposed along the temporal sclera. To block the optic nerve and lead to a crush injury of the optic nerve, a conical soft silica gel with a $2 \mathrm{~mm}$ diameter at the thin end was tucked in the optic hole. Two days after surgery, rabbits with pupils $>4.5 \mathrm{~mm}$, with disappearance of direct light reflex and no clear extruded eyeballs were regarded as success models. Optic nerve decompression surgery was carried out to remove the silica gel (2).

Pattern reversal visual evoked potentials (P-VEP). All the examinations were performed using a NeuroMax 1004 machine (Xltek, Oakville, ON, Canada) in a dark room at $18-25^{\circ} \mathrm{C}$, with all ketamine rabbits anesthetized. Recording electrodes were placed $\sim 5 \mathrm{~cm}$ away and back from the midpoint of the ears, the reference electrode was subcutaneously placed at the midpoint of the eyes, and the ground electrode was placed at the tip of the ipsilateral ear. The eyes were $30 \mathrm{~cm}$ away from the screen, to flip stimulate (alternate light and shade of the stimulus) the full-field monocular vision with an all wild checkerboard pattern, whilst the contralateral eye was masked. Stimulation parameters were as follows: The checkerboard was of size 30' (half light and half shade), with an average luminance $50 \mathrm{CD} / \mathrm{m}^{2}, 90 \%$ contrast, and the flip frequency was $1.1 \mathrm{~Hz}$. Stimulus-caused signals were filtered (with a passband of $0.5-100 \mathrm{~Hz})$, amplified ( $2 \times 10^{4}$ times) and input onto a microcomputer for superposition. Superposition was for $128 \mathrm{x}$, and analysis for $300 \mathrm{msec}$. The P-VEP waveforms of $1 \mathrm{~h}$ pre- and post-trauma, $1 \mathrm{~h}$ pre-decompression, and 2 weeks post-decompression were recorded, and the latency and amplitude of the main wave (P-wave) were determined, with each stimulus repeated $\geq 3 x$. For the normal group, a set of data were collected only as a control, and data of the non-decompression group were collected in accordance of the check time of the 2 weeks decompression group. The data and waveform were collected and statistical processed by a microcomputer.

The P-VEP observation indexes included the absolute incubation period and amplitude of the NPN contours main wave (P-wave). The absolute incubation period is the time from the beginning of stimulation to the peak of the P-wave, and the irregular P-wave shape can be measured from the intersection of an extension cord of the rise and fall branch of the P-wave peak. Absolute volatility is the range from a previous negative peak to the amplitude of the P-wave. The latent period and amplitude mentioned in the following are all absolute incubation period and amplitude.

Optic nerve morphology. All the experimental animals of the post-trauma decompression group were sacrificed 2 weeks after the decompression, and the optic nerves were fixed in $10 \%$ formaldehyde solution, stained with haematoxylin and eosin, and observed under a light microscope. The non-decompression group was sacrificed 4 weeks after trauma, with the same treatment as before. The optic nerve specimens in the normal control group were collected as the control. Morphological observations included swelling of the optic nerve, axonal degeneration, myelin depigmentation, hyperplasia of astrocytes, and vascular lesions within the optic nerve.
Statistical analysis. The data are presented as the mean \pm standard deviation. All statistical analyses were performed using SPSS 10.0 (SPSS, Inc., Chicago, IL, USA). Independent sample t-test was used in self-comparison of pre- and postdecompression, and one-way analysis of variance was used to compare the P-VEP of multiple groups 2 weeks post-decompression. Differences between groups were compared using the least significant difference test when homoscedasticity was assumed. $\mathrm{P}<0.05$ was considered to indicate a statistically significant difference.

\section{Results}

$P$-VEP changes. The P-VEP of each healthy rabbit revealed typical NPN contours. The NPN contours in the injured rabbits were low and flat. The latent period of the $\mathrm{P}$ contour was lengthened and the amplitude was reduced (Tables I and II). The differences between the latent period and amplitude preand post-trauma were significant statistically $(\mathrm{P}<0.05)$. The comparisons between P-VEP pre- and post-decompression were as follows: Comparisons of the amplitude and latent periods prior and subsequent to decompression in the i) 48-h decompression group were significantly different $(\mathrm{P}<0.05)$, ii) in the 1-week decompression group exhibited no differences ( $P>0.05)$, and iii) comparisons of amplitude prior and subsequent to decompression in the 2-week decompression group exhibited no differences $(\mathrm{P}>0.05)$, while the latent period was significantly different $(\mathrm{P}<0.05)$.

The comparisons of P-VEP 2 weeks post-decompression were as follows: i) The differences of the latent period between two groups among B, C and D were significantly different $(\mathrm{P}<0.01)$, those of amplitude between groups $\mathrm{B}$ against $\mathrm{C}$ and $\mathrm{D}$ were significant $(\mathrm{P}<0.01)$, while those between groups $\mathrm{C}$ and $\mathrm{D}$ were significant $(\mathrm{P}<0.05)$. ii) The differences of the latent period and amplitude between groups $\mathrm{B}$ and $\mathrm{C}$ with group $\mathrm{E}$ were significant $(\mathrm{P}<0.01)$, while those between groups $\mathrm{D}$ and $\mathrm{E}$ exhibited no significance $(\mathrm{P}>0.05)$. iii) There was no significance in the differences of amplitude and latent period between groups $\mathrm{A}$ and $\mathrm{B}(\mathrm{P}>0.05)$, while there were significant differences among group $\mathrm{A}$ and groups $\mathrm{C}-\mathrm{E}$ $(\mathrm{P}<0.01)$.

Optic nerve morphological changes. In the normal control group, the astrocytes of the optic nerve exhibited a cylindrical form and were arranged evenly on vertical section (Fig. 1) the neural fibers were arranged neatly and were stained evenly, and the cross section showed a normal configuration of the blood vessel.

For the 48-h decompression group, the arrangement of the astrocytes was even on the vertical section, with vacuoles, slight swelling of the nerve, exudation around the blood vessel and a small amount of astrocytes hyperplasia observed in the damaged area (Fig. 2).

In the 1-week group, the arrangement of the astrocytes was inordinate on the vertical section. Numerous vacuoles of different sizes, demyelination of the nerve, uncovered axons, clear exudation around the blood vessel and evident astrocytes hyperplasia were observed in the majority of areas.

In the 2-week group, the cylindrical arrangement of the astrocytes disappeared completely on the vertical section. 
Table I. Latent period of the P-wave in the different periods.

\begin{tabular}{lcccc}
\hline Groups & $\begin{array}{c}\text { Pre-trauma, } \\
\text { msec }\end{array}$ & $\begin{array}{c}1 \text { h post-trauma, } \\
\text { msec }\end{array}$ & $\begin{array}{c}\text { 1 h pre-decompression, } \\
\text { msec }\end{array}$ & $\begin{array}{c}2 \text { weeks post-decompression, } \\
\text { msec }\end{array}$ \\
\hline A & $63.67 \pm 4.74$ & NA & NA & NA \\
B & $62.03 \pm 8.79$ & $71.77 \pm 7.96$ & $86.47 \pm 14.28$ & $71.25 \pm 8.51$ \\
C & $65.91 \pm 12.78$ & $80.58 \pm 9.38$ & $94.72 \pm 7.13$ & $106.43 \pm 11.79$ \\
D & $63.50 \pm 7.69$ & $74.02 \pm 6.14$ & $116.35 \pm 17.13$ & $158.73 \pm 15.16$ \\
$E^{a}$ & $63.80 \pm 7.60$ & $75.85 \pm 5.75$ & $114.42 \pm 19.57$ & $169.75 \pm 16.25$ \\
\hline
\end{tabular}

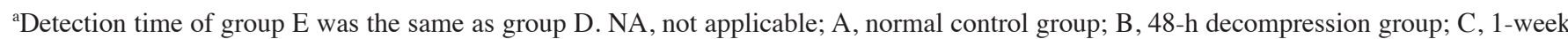
decompression group; D, 2-week decompression group; E, non-decompression group.

Table II. Amplitude of the P-wave in the different periods.

\begin{tabular}{lcccc}
\hline Groups & $\begin{array}{c}\text { Pre-trauma, } \\
\mu \mathrm{V}\end{array}$ & $\begin{array}{c}1 \mathrm{~h} \text { post-trauma, } \\
\mu \mathrm{V}\end{array}$ & $\begin{array}{c}\text { 1 h pre-decompression, } \\
\mu \mathrm{V}\end{array}$ & $\begin{array}{c}2 \text { weeks post-decompression, } \\
\mu \mathrm{V}\end{array}$ \\
\hline $\mathrm{A}$ & $5.35 \pm 1.17$ & $\mathrm{NA}$ & $\mathrm{NA}$ & $\mathrm{NA}$ \\
$\mathrm{B}$ & $5.93 \pm 1.09$ & $4.82 \pm 0.50$ & $4.42 \pm 0.42$ & $5.25 \pm 0.78$ \\
$\mathrm{C}$ & $5.58 \pm 1.47$ & $4.05 \pm 0.82$ & $3.05 \pm 0.71$ & $2.88 \pm 0.76$ \\
$\mathrm{D}$ & $5.00 \pm 0.85$ & $3.85 \pm 0.62$ & $2.53 \pm 0.78$ & $1.83 \pm 0.96$ \\
$\mathrm{E}^{\mathrm{a}}$ & $5.31 \pm 0.83$ & $4.20 \pm 0.81$ & $2.60 \pm 0.92$ & $1.58 \pm 0.63$ \\
\hline
\end{tabular}

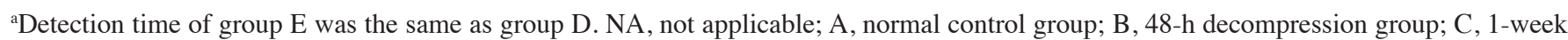
decompression group; D, 2-week decompression group; E, non-decompression group.

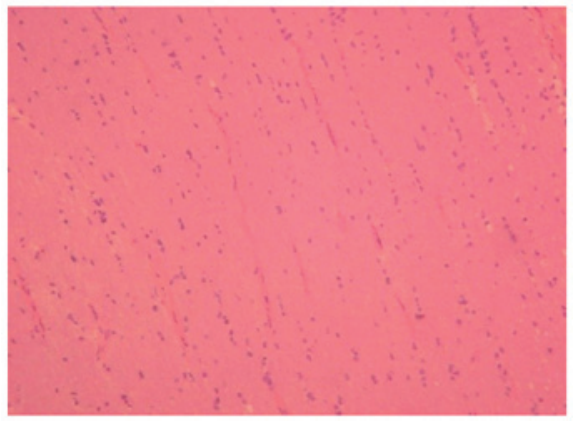

Figure 1. Normal optic nerve. The vertical section shows that astrocytes are arranged evenly, and the neural fibers arranged neatly and were evenly stained (haematoxylin and eosin; magnification, x100).

Evident astrocytic hyperplasia filled the field of view, and clear nerve demyelination and local necrosis was observed, while exudation around the blood vessel was reduced, with inflammatory infiltration in some of the blood vessels (Fig. 3).

In the non-decompression group, large areas of necrosis, evident nerve demyelination, serious exudation around the blood vessel and astrocytic hyperplasia were observed.

\section{Discussion}

Optic nerve injury is a type of severe head trauma combined injury, and numerous studies have established a series of optic nerve injury animal models, such as optic nerve transverse,

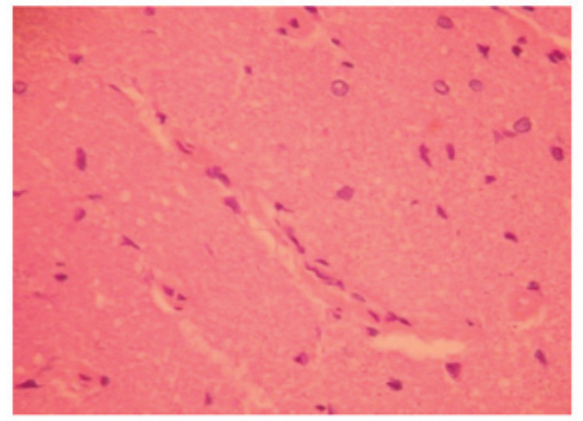

Figure 2. Optic nerve of the 48-h decompression group. The arrangement of the astrocytes was even on the vertical section, and vacuoles were observed in the damaged area (haematoxylin and eosin; magnification, x200).

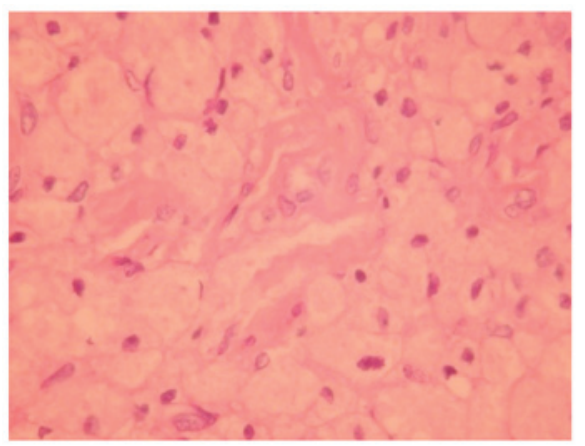

Figure 3. Optic nerve of the 2-week decompression group. The cylindrical arrangement of astrocytes disappeared completely and local necrosis was apparent on the vertical section (haematoxylin and eosin; magnification, x200). 
tractive and crush injury (3-5); each has its advantages and disadvantages. Optic nerve transection injury is established by cutting off the optic nerve at any position prior to the optic chiasma, leading to all retinal optic ganglion cell axons to rupture completely, which can guarantee all experimental animals at the same degree of wounding and is convenient for a control study. The common characteristics of the optic nerve crush animal model are to crush the optic nerve at any position prior to optic chiasma with different instruments, to maintain the integrity of the epineurium. This model is simple, easy to operate, and can exactly establish the optic nerve injury with a high injury rate, as exposure of the optic nerve from the conjunctival approach results in lighter trauma to the experimental animals, with a low animal mortality, improved repeatability, and is similar to clinical practice, therefore, it is widely used. However, the injury degree is operator dependent, as the optic nerve damage is variable under different operators, and the damage degree is difficult to quantitate precisely. The optic nerve tractive injury animal model includes parallel and vertical optic canal axial traction. The former can exactly lead to diffuse axonal injury, the latter is similar to optic canal fracture caused by a cutting injury. The optic nerve crush injury animal model can imitate the optic nerve damage that extrudes the surrounding tissue of the orbital neoplasm and clinical indirect optic nerve injury. This simple surgery is easy to operate, can cause exact optic nerve damage in experimental animals with less trauma, and postoperative nursing and further observation is simple. All the aforementioned models have limitations on quantifying the injuries. In the present study, a circular cone soft silicone was adopted to block the optic nerve to establish an optic nerve crush injury, and the size of the smaller end and the depth of extrusion were controlled. The P-VEP and pathological results showed that the optic nerve crush injury was incomplete, and the injury degree was consistent.

Clinically, indirect optic nerve injury caused by head trauma is difficult to examine and evaluate. Visual electrophysiological examination is an objective visual function assessment method, and can clinically improve early diagnosis ability of indirect optic nerve injury $(6,7)$. VEP can develop from visual information through the visual nerve system. At the later stages of visual formation are the mass responses to visual stimuli that are recorded from electrodes placed on the cerebral cortex, which can reflect the total functional status from the retinal ganglion cells to the visual cortex. VEP, mainly on behalf of the electrical activity complicated within the process of visual production in the $10-20^{\circ}$ of the central vision, from the synapse, axon, optic nerve to the occipital visual cortex, can sensitively reflect the integrity and function of the neuron axon and myelin sheath in each optic nerve district. It has been shown that following all wild stimulation in one eye of the rabbit, the occurrence rate of the P-wave in the NPN contours recorded from the cortex was $100 \%$, and the latent period was the most stable, with a higher amplitude and relatively variable $\mathrm{N}$-wave (8). Therefore, in the present experiment, only the wave latency and amplitude of the P-wave were used for analysis. There are numerous types of visual evoked cortical potential. As a stable waveform with small individual variation, P-VEP is widely used to evaluate the optic nerve diseases. In optic nerve tractional injury, Bain et al (9) reported that the electrophysiological changes began to appear when the optic nerve stretch to $5.5 \mathrm{~mm}$, while the morphology change appeared following a stretch to $6.8 \mathrm{~mm}$, suggesting that electrophysiological changes are more sensitive compared to morphology. Therefore, P-VEP was used as the main observation index in the present study.

Compared with the normal control group, the incubation period of the P-wave was evidently prolonged in the early stage of trauma, with a significantly lower amplitude, suggesting that visual function is not completely lost in the direct mechanical effect caused partly by axon injury. Subsequently, the incubation period continued to prolong, and the amplitude continued to reduce. Following direct injury, the blood circulation obstacle and tissue edema of the surrounding environment caused secondary axonal injury leading to the further decline in visual function. The 2-week post-decompression P-VEP results showed that when decompression was implemented 48 -h after injury, the incubation period will be significantly shorter compared to pre-decompression, and can return to normal levels, with no evident difference to group A. When decompression occurs 1 or 2 weeks after injury, there were no evident improvements on the incubation period, and the incubation period of the 2-week post-decompression group was markedly prolonged compared to the 2 -week pre-decompression. These results indicate that following optic nerve injury, early decompression can protect the visual function better than later decompression, and to a certain extent it can reverse the damage on visual function, as early decompression (1 week) can prevent axon secondary injury, avoiding further visual function decline, whereas decompression 2 weeks after is not ineffective. From 108 cases of clinical superciliary arch fractures, Matteini et al (1) concluded that the best surgical intervention must be carried out within 7 days, and $<12$ days, which is consistent with the present experimental results.

Swelling, myelinoclasis and axon changes were the main observations following indirect optic nerve injury. In the present experiment, due to the difference of decompression time after injury, the optic nerve morphology change was different at 2 weeks after decompression. In the 48-h decompression group, vacuoles, slight swelling of the nerve, exudation around the blood vessel and a small amount of astrocytes hyperplasia were observed in the damaged area, whereas the arrangement of astrocytes was even on the vertical section. As the decompression time prolonged, myelinoclasis aggravated gradually, accompanied by necrosis of the damaged area, and inordinate arrangement and evident hyperplasia of the astrocytes. The present results showed that as the decompression time prolonged, the optic nerve crush injury appeared to exhibit mild to severe local pathological changes, and evident myelinoclasis changes and astrocytes proliferation appeared in the non-decompression trauma group 4 weeks after injury. Certain studies $(10,11)$ quantitatively analyzed retinal optic ganglion cells (RGCs) following optic nerve crush injury and reported that RGCs reduced rapidly in the first 2 weeks, reduce to $\sim 50 \%$ at the end of 2 weeks, and decreased slowly at the latter 2 weeks. Thus, direct optic nerve damage can lead to part of the axon injury and induce a series of changes, such as RGC acute loss, impaired RGC release cytotoxic compounds, causing intracellular calcium overload, free radicals and 
glutamic acid increase, followed by an intact axon or the original RGCs on the damage edge in axons suffered from secondary degeneration (12). Therefore, positive and effective measures following optic nerve injury can reduce and delay the secondary injury of RGCs, otherwise, the RGCs will eventually degenerate (13).

The pathogenesis of traumatic optic nerve injury remains to be elucidated. Maxwell et al (14) proposed that due to space limitations of the optic canal, progressive blood circulation disorder and hematoma of the indirect optic nerve injury may turn into a vicious cycle, which leads to further loss of the original recovery potential of the injured optic fiber. The optic nerve secondary damage theories are the theoretical basis of optic nerve decompression. There are controversies on the treatment of traumatic optic nerve injury, and the choice of clinical treatment is largely based on personal experience (15). Goldberg and Steinsapir (16) proposed that optic nerve decompression is beneficial to the recovery of indirect optic nerve injury regardless of whether there are optic canal fractures. Cook et al (17) retrospectively analyzed the treatment results of 244 patients reported in 46 studies, and identified that the treatment group recovered better than the no treatment group. No significant difference was apparent between the curative effect of surgical decompression, hormone therapy and surgery plus hormone treatment, and visual recovery was associated with the extent of the initial injury. Recovery of patients without fractures was better than with fractures, and anterior ethmoid fracture was better than posterior ethmoid fracture.

The present study established a rabbit optic nerve crush injury animal model, and systematically observed visual function changes following optic nerve injury in the different periods after decompression. Neuron secondary injury is one of the main reasons for the progressive decline of the optic nerve function, and therefore, protecting neurons without primary damage from secondary damage is an important aspect of optic nerve injury research. Optic nerve decompression can reduce the indirect optic nerve injury, and early decompression following optic nerve injury can better protect the visual function compared to later decompression. To a certain extent, this can avoid further decline of visual function and reverse optic nerve function damage (18).

\section{Acknowledgements}

The authors would like to thank Dr Y.L. Cao from the Medical School of Nanjing University for providing the animal model. The present study was supported by a grant (no. CNJ13C005) from the Medical Science Foundation of Chinese People's Liberation Army.

\section{References}

1. Matteini C, Renzi G, Becelli R, Belli E and Iannetti G: Surgical timing in orbital fracture treatment: Experience with 108 consecutive cases. J Craniofac Surg 15: 145-150, 2004.

2. Feng Y, Luo L, Ma Z, Sun X and Hu Y: In vivo detection of severity of optic nerve crush using manganese-enhanced magnetic resonance imaging in rats. Chin Med J (Engl) 127: 522-527, 2014.

3. Solomon AS, Lavie V, Hauben U, Monsonego A, Yoles E and Schwartz M: Complete transection of rat optic nerve while sparing the meninges and the vasculature: An experimental model for optic nerve neuropathy and trauma. J Neurosci Methods 70: 21-25, 1996.

4. Bien A, Seidenbecher CI, Böckers TM, Sabel BA and Kreutz MR: Apoptotic versus necrotic characteristics of retinal ganglion cell death after partial optic nerve injury. J Neurotrauma 16: 153-163, 1999.

5. Yoles E, Wheeler LA and Schwartz M: Alpha2-adrenoreceptor agonists are neuroprotective in a rat model of optic nerve degeneration. Invest Ophthalmol Vis Sci 40: 65-73, 1999.

6. Nakamura A, Akio T, Matsuda E and Wakami Y: Pattern visual evoked potentials in malingering. J Neuroophthalmol 21: 42-45, 2001.

7. Yan H, Li F and Zhang L: A new and reliable animal model for optic nerve injury. Curr Eye Res 37: 941-948, 2012.

8. Polianskiı̌ VB, Evtikhin DV and Sokolov EN: The brightness components of the visual evoked potential to color stimuli in the rabbit. Zh Vyssh Nerv Deiat Im I P Pavlova 49: 1046-1051, 1999 (In Russian).

9. Bain AC, Raghupathi R and Meaney DF: Dynamic stretch correlates to both morphological abnormalities and electrophysiological impairment in a model of traumatic axonal injury. J Neurotrauma 18: 499-511, 2001.

10. Takano M: Axonal regeneration of retinal ganglion cells. Nippon Ganka Gakkai Zasshi 100: 972-981, 1996 (In Japanese).

11. Lynch DR and Dawson TM: Secondary mechanisms in neuronal trauma. Curr Opin Neurol 7: 510-516, 1994.

12. Povlishock JT and Christman CW: The pathobiology of traumatically induced axonal injury in animals and humans: A review of current thoughts. J Neurotrauma 12: 555-564, 1995.

13. Yoles E and Muller S: NMDA-receptor antagonist protects neurons from secondary degeneration after partial optic crush. J Neurotrauma 16: 153-163, 1995.

14. Maxwell WL, Povlishock JT and Graham DL: A mechanistic analysis of nondisruptive axonal injury: A review. J Neurotrauma 14: 419-440, 1997.

15. Zimmerer R, Rana M, Schumann P and Gellrich NC: Diagnosis and treatment of optic nerve trauma. Facial Plast Surg 30: 518-527, 2014.

16. Goldberg RA and Steinsapir KD: Extracranial optic canal decompression: Indications and technique. Ophthal Plast Reconstr Surg 12: 163-170, 1996.

17. Cook MW, Levin LA, Joseph MP and Pinczower EF: Traumatic optic neuropathy. A meta-analysis. Arch Otolaryngol Head Neck Surg 122: 389-392, 1996.

18. Chen F, Zuo K, Feng S, Guo J, Fan Y, Shi J and Li H: A modified surgical procedure for endoscopic optic nerve decompression for the treatment of traumatic optic neuropathy. N Am J Med Sci 6: 270-273, 2014. 